\title{
Tooth size changes with age in a Spanish population: Percentile tables
}

\author{
Vera Paulino ${ }^{1}$, Vanessa Paredes ${ }^{2}$, Rosa Cibrian ${ }^{3}$, José-Luis Gandia ${ }^{4}$
}

${ }^{1} \mathrm{PhD}$ in Dentistry. MS in Orthodontics. Private practice of orthodontics, Lisbon, Portugal. Collaborate Professor. Department of Orthodontics. Faculty of Medicine and Dentistry, University of Valencia, Spain

${ }^{2} \mathrm{PhD}$ in Dentistry. MS in Orthodontics. Doctor Professor. Department of Orthodontics. Faculty of Medicine and Dentistry, University of Valencia, Spain

${ }^{3}$ Professor. Department of Physiology. Faculty of Medicine and Dentistry, University of Valencia, Spain

${ }^{4}$ Professor. Director of Postgraduate Orthodontics Masters Course. Department of Orthodontics. Faculty of Medicine and Dentistry, University of Valencia, Spain

Correspondence:

Unidad docente de Ortodoncia.

Clínica Odontológica.

C/ Gascó Oliag $n^{\circ} 1$.

Valencia 46010. Spain

verapaulino@hotmail.com

Paulino V, Paredes V, Cibrian R, Gandia JL. Tooth size changes with age in a Spanish population: Percentile tables. Med Oral Patol Oral Cir Bucal. 2011 Sep 1;16 (6):e840-4

http://www.medicinaoral.com/medoralfree01/v16i6/medoralv16i6p840.pdf

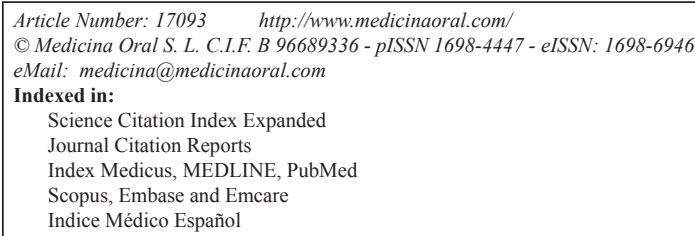

\begin{abstract}
Objectives: The aims of this work were: firstly, to draw up tables of percentile tooth sizes similar to those of Sanin and Savara for three age groups of a Spanish population; secondly, to describe changes in tooth size between those groups over time, as well as observing any sexual dimorphism and, finally, to compare both the Spanish and Sanin and Savara's American population samples.

Material and methods: The sample included 359 patients and was divided into three age groups: adolescents, young adults and adults, of both genders. After dental cast digitalization, mesiodistal tooth-size was measured on each dental cast using a digital method. Dental size tables organized by percentiles for each group of age and gender were drawn up. Percentiles under 30 were considered as small, between 30 and 70 as average, and above 70 as large. As symmetry was found between contralateral teeth, the mean between the teeth of the two semi-arches was considered.

Results: The mesiodistal tooth sizes of adolescents did not present statistically significant differences between genders, in contrast to the two other age groups.

Conclusions: Mesiodistal tooth diameters tended to diminish with age, especially in women, in the Spanish population. The values obtained for our dental tables, organized by percentiles, were slightly higher than those found by Sanin and Savara in an American population, especially for women.
\end{abstract}

Key words: Mesiodistal tooth size, mesiodistal tooth diameter, dental size tables, percentiles. 


\section{Introduction}

Determining mesiodistal tooth size is a fundamental tool for orthodontic diagnosis, as mesiodistal tooth size is related with several malocclusions, such as dental crowding (1). Moreover, sexual dimorphism is observed in the mesiodistal size of teeth in most studies, women generally presenting smaller sizes, regardless of ethnic group $(2,3)$.

Many authors (2,3-6) have drawn up tooth-size tables in order to classify tooth sizes in accordance with the ethnic group and type of malocclusion. However, Sanin and Savara (7), with the aim of establishing a standard for locating and analysing tooth-size discrepancies have been the only ones so far, to develop tables organised into percentiles of $10 \mathrm{~s}$, from a minimum percentile below 10 to another maximum above 90 . In their study they selected, from the Child Study Clinic of the University of Oregon Dental School, 51 American males and 50 American females of European ancestry with permanent dentition and presented different tables for both genders. The teeth situated in the percentiles up to 30 were considered as small, those situated between percentiles 30 and 70 as average-sized teeth, and those in percentiles greater than 70 as large teeth. Using this table, organized by percentiles, we can evaluate whether the maxillar and mandibular teeth are proportional to each other, whether either of them is out of line with the others, the direction of discrepancy (small, medium, large) and its magnitude (difference in percentiles).

Taking into account that there are no dental size percentile tables on the Spanish population in the literature, the objectives of the present work were:

1- To draw up tables of percentile tooth sizes similar to those of Sanin and Savara for each of the three age groups (adolescents, young adults and adults) of a Spanish population and to describe changes in tooth size over time between those groups, as well as to observe any sexual dimorphism.

2- To compare tooth sizes of both populations: the Spanish and the American Caucasian (Sanin and Savara) for the adolescent group.

\section{Materials and Methods}

The sample was selected from patients attending the Orthodontic Department of the University of Valencia, Spain. It was made up of individuals of both genders, all of them Valencian, resident in the city of Valencia, Spain, and with Valencian parents. The sample was homogeneous and was divided into 3 chronological age groups: adolescents, young adults and adults.

The final sample consisted of 359 individuals:

1) 99 adolescents (65 females and 34 males) with a mean age of 14.15 years (age range: 11-17 years).

2) 110 young adults (53 females and 57 males) with a mean age of 21.9 years (age range: 19-26 years).
3) 150 adults ( 72 females and 78 males) with a mean age of 40 years (age range: 31-50 years).

All three types of Angle molar class were present in all groups and none of the subjects had previously received orthodontic treatment.

The material used in this study consisted of:

1. Plaster casts.

2. A conventional scanner for digitalising all study models.

3. Our own technology program, developed at our department, whose reliability and reproducibility in determining mesiodistal tooth size had previously been tested (8). This program requires both a digitalised image of what we wish to measure and back-up software for undertaking measurements.

The inclusion criteria for the study models were as follows:

1. Presence of permanent dentition from first left-side molar to first right-side molar

2. Absence of alteration in the number of teeth.

3. Absence of abnormalities in dental size and shape.

4. Good quality of study models.

On each of the digitalised plaster casts, mesiodistal tooth size was measured as the maximum distance between contact points on proximal surfaces, both mesial and distal. For malpositioned teeth the hypothetical contact points were measured on their proximal surfaces. Second and third molars were excluded from measurements.

\section{-Statistical method}

The values obtained were entered into a database to be processed using the Statistical Package for Social Sciences version 15 for Windows (SPSS Inc., v.15®, Chicago, Illinois, USA).

In all cases, normal distribution could be checked using the Kolmogorov-Smirnov test, with a probability of $p$ no lower than 0.395 . Despite the evident symmetry between the right and left semi-arches, we calculated the mean differences between each pair of teeth, right to left, observing no differences greater than $0.02 \mathrm{~mm}$. This is in line with the technique's own margin of measurement error, all correlations between collateral teeth being greater than 0.930 . The mean between both semiarches was, therefore, considered for each tooth.

A 2-factor variance analysis was undertaken in order to compare the mean mesiodistal sizes between genders and age-groups using the Bonferroni test for gender and the Scheffé test for age groups.

For each of the three groups analysed, tables of mesiodistal tooth sizes, ordered by percentiles, were determined. 


\section{Results}

The 2-factor variance analysis indicated that both gender $(p<0.01)$ and age $(p<0.001)$ were factors that had an influence on mesiodistal size, but whereas for young adults and adults the difference per gender was significant ( $p<0.01$ and $p<0.02$ respectively), in the adolescent group a strong similarity in the mean values of each tooth between the genders was observed. As no significant statistical difference exists between the values ob- tained, it would have been sufficient to present a single table. However and despite that, we have presented the mesiodistal tooth-size tables, ordered by percentiles and separated by gender for all age groups: adolescents, young adults and adults (Tables 1, 2 and 3).

Figure 1 is presented as an example of comparing tooth sizes from our sample with those of Sanin and Savara's for the minimum $(0)$, medium $(50,60)$ and maximum (100) percentiles.
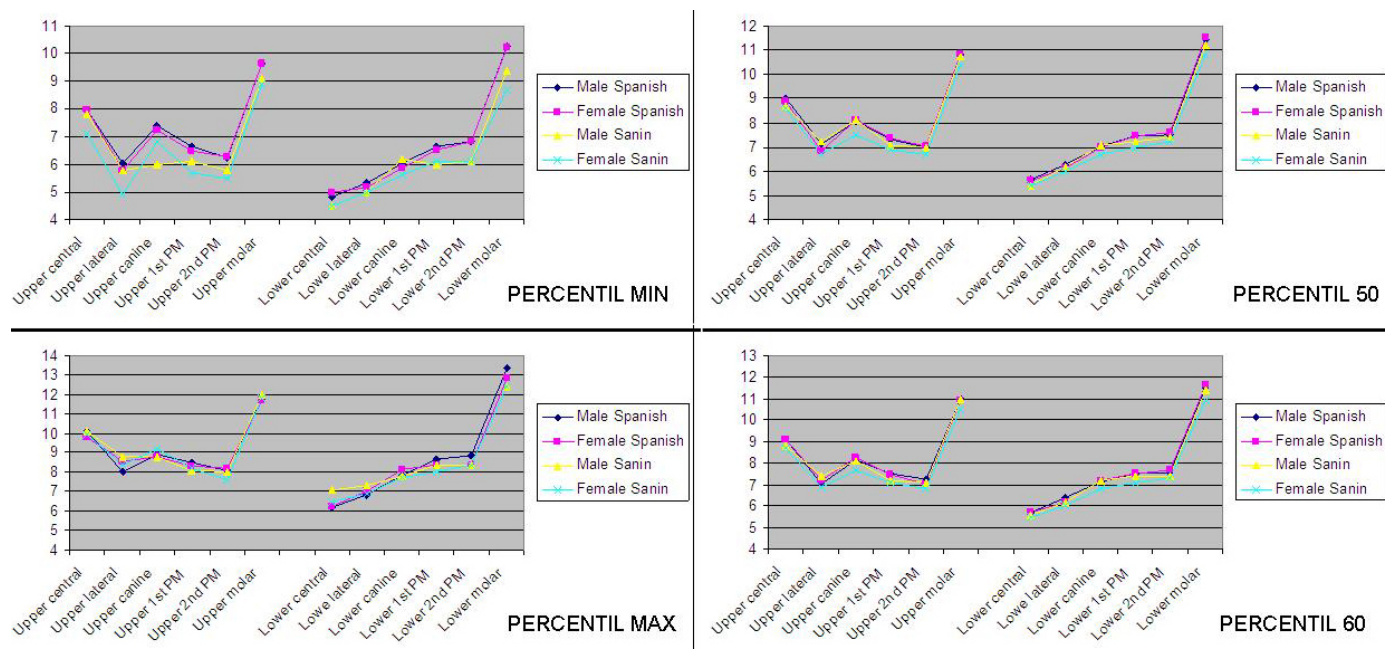

Fig. 1. Comparison of tooth size of our sample with those of Sanin and Savara's to the minimum (0), medium $(50,60)$ and maximum (100) percentiles for both genders and for each tooth: central (central incisor); lateral (lateral incisor); canine; 1stPM (first premolar); 2nd PM (second premolar) and molar ( first molar) of the upper and lower arch.

Table 1. Mesiodistal tooth-size table (percentiles) for adolescents (male-M and female-F) for both maxillary (MX) and mandibular (MB) arches. CI-central incisor; LI-lateral incisor; C-canine; 1PM-1rst premolar; 2PM-2nd premolar; 1M-1rst molar.

\begin{tabular}{|c|c|c|c|c|c|c|c|c|c|c|c|c|c|c|c|c|c|c|c|c|c|c|}
\hline \multirow{3}{*}{ TEETH } & \multicolumn{6}{|c|}{ Small } & \multicolumn{10}{|c|}{ Average } & \multicolumn{6}{|c|}{ Large } \\
\hline & \multicolumn{2}{|c|}{ Min. } & \multicolumn{2}{|c|}{10} & \multicolumn{2}{|c|}{20} & \multicolumn{2}{|c|}{30} & \multicolumn{2}{|c|}{40} & \multicolumn{2}{|c|}{50} & \multicolumn{2}{|c|}{60} & \multicolumn{2}{|c|}{70} & \multicolumn{2}{|c|}{80} & \multicolumn{2}{|c|}{90} & \multicolumn{2}{|c|}{ Máx. } \\
\hline & \begin{tabular}{ll|}
$\mathrm{M}$ \\
\end{tabular} & $\mathrm{F}$ & $\mathrm{M}$ & $\mathrm{F}$ & $\mathrm{M}$ & $F$ & $\mathrm{M}$ & $\mathrm{F}$ & $\mathrm{M}$ & $\mathrm{F}$ & $\mathrm{M}$ & $F$ & M & $\mathrm{F}$ & $\mathrm{M}$ & $\mathrm{F}$ & \begin{tabular}{l|l}
$\mathrm{M}$ & \\
\end{tabular} & $\mathrm{F}$ & \begin{tabular}{l|l|l|} 
M & \\
\end{tabular} & $F$ & \begin{tabular}{l|l|l|} 
M & \\
\end{tabular} & $\mathrm{F}$ \\
\hline \multicolumn{23}{|c|}{ MX Arch } \\
\hline CI & 7,98 & 7,96 & 8,24 & 8,12 & 8,46 & 8,24 & 8,75 & 8,38 & 8,90 & 8,65 & 9,01 & 8,86 & 9,10 & 9,09 & 9,29 & 9,20 & 9,47 & 9,36 & 9,70 & 9,65 & 10,12 & 9,79 \\
\hline 니 & 6,01 & 5,78 & 6,38 & 6,42 & 6,68 & 6,46 & 6,97 & 6,55 & 7,02 & 6,69 & 7,11 & 6,84 & 7,06 & 7,19 & 7,33 & 7,28 & 7,48 & 7,73 & 7,62 & 8,21 & 8,00 & 8,57 \\
\hline c & 7,39 & 7,25 & 7,41 & 7,48 & 7,72 & 7,64 & 7,81 & 7,80 & 7,96 & 7,98 & 8,05 & 8,12 & 8,15 & 8,25 & 8,28 & 8,40 & 8,50 & 8,42 & $8,67 \mid$ & 8,72 & 8,94 & 8,81 \\
\hline 1PM & 6,64 & 6,50 & 6,80 & 6,85 & 7,06 & 6,96 & 7,15 & 7,13 & 7,24 & 7,25 & 7,31 & 7,38 & 7,50 & 7,44 & 7,54 & 7,54 & 7,77 & 7,76 & 7,95 & 8,05 & 8,51 & 8,30 \\
\hline 2PM & 6,23 & 6,27 & 6,52 & 6,52 & 6,66 & 6,69 & 6,73 & 6,84 & 6,88 & 6,92 & 6,97 & 7,04 & 7,23 & 7,04 & 7,34 & 7,09 & 7,23 & 7,47 & 7,40 & 7,60 & 8,09 & 8,20 \\
\hline $1 \mathrm{M}$ & 9,64 & 9,65 & 10,04 & 9,99 & 10,27 & 10,30 & 10,44 & 10,44 & 10,60 & 10,62 & 10,80 & 10,82 & 10,95 & 10,94 & 11,10 & 11,04 & 11,16 & 11,25 & 11,43 & 11,55 & 11,74 & 11,70 \\
\hline \multicolumn{23}{|c|}{ MB Arch } \\
\hline CI & 4,83 & 4,99 & 5,15 & 5,09 & 5,39 & 5,19 & 5,49 & 5,41 & 5,56 & 5,53 & 5,65 & 5,59 & 5,73 & 5,73 & 5,86 & 5,85 & 6,11 & 5,95 & 6,14 & 6,01 & 6,19 & 6,23 \\
\hline 니 & 5,33 & 5,14 & 5,53 & 5,49 & 5,87 & 5,61 & 6,03 & 5,83 & 6,02 & 6,14 & 6,26 & 6,11 & 6,40 & 6,19 & 6,31 & 6,45 & 6,51 & 6,56 & 6,72 & 6,71 & 6,83 & 7,02 \\
\hline C & 6,05 & 5,88 & 6,61 & 6,44 & 6,74 & 6,59 & 6,90 & 6,69 & 6,85 & 6,99 & 7,03 & 6,98 & 7,12 & 7,20 & 7,33 & 7,23 & $\mathbf{7 , 4 2}$ & 7,34 & 7,57 & 7,52 & 7,82 & 8,16 \\
\hline 1PM & 6,65 & 6,52 & 6,91 & 6,84 & 7,12 & 7,18 & 7,21 & 7,25 & 7,38 & 7,35 & 7,46 & 7,46 & 7,53 & 7,53 & 7,64 & 7,66 & 7,76 & 7,76 & 7,94 & 7,97 & 8,65 & 8,37 \\
\hline 2PM & 6,82 & 6,81 & 6,98 & 6,92 & 7,09 & 7,13 & 7,32 & 7,27 & 7,42 & 7,39 & 7,48 & 7,61 & 7,58 & 7,70 & 7,85 & 7,76 & 7,99 & 7,83 & 8,32 & 8,04 & 8,88 & 8,40 \\
\hline $1 \mathrm{M}$ & 10,24 & 10,20 & 10,61 & 10,48 & 10,81 & 10,72 & 11,04 & 11,21 & 11,46 & 11,23 & 11,41 & 11,51 & 11,51 & 11,64 & 11,94 & 11,62 & 12,05 & 11,79 & 12,20 & 12,03 & 13,34 & 12,81 \\
\hline
\end{tabular}


Table 2. Mesiodistal tooth-size table (percentiles) for young adults (male-M and female-F) for both maxillary (MX) and mandibular (MB) arches. CI-central incisor; LI-lateral incisor; C-canine; 1PM-1rst premolar; 2PM-2nd premolar; 1M-1rst molar.

\begin{tabular}{|c|c|c|c|c|c|c|c|c|c|c|c|c|c|c|c|c|c|c|c|c|c|c|}
\hline \multirow{3}{*}{ TEETH } & \multicolumn{6}{|c|}{ Small } & \multicolumn{10}{|c|}{ Average } & \multicolumn{6}{|c|}{ Large } \\
\hline & \multicolumn{2}{|c|}{ Min. } & \multicolumn{2}{|c|}{10} & \multicolumn{2}{|c|}{20} & \multicolumn{2}{|c|}{30} & \multicolumn{2}{|c|}{40} & \multicolumn{2}{|c|}{50} & \multicolumn{2}{|c|}{60} & \multicolumn{2}{|c|}{70} & \multicolumn{2}{|c|}{80} & \multicolumn{2}{|c|}{90} & \multicolumn{2}{|c|}{ Máx. } \\
\hline & M & $F$ & M & $F$ & M & $\mathrm{F}$ & $M$ & $F$ & $\mathrm{M}$ & $F$ & M & $F$ & $\mathrm{M}$ & $\mathrm{F}$ & $\mathrm{M}$ & $F$ & $M$ & $F$ & M & $F$ & M & $F$ \\
\hline \multicolumn{23}{|c|}{ Mx Arch } \\
\hline $\mathrm{Cl}$ & 7,73 & 6,93 & 8,02 & 7,87 & 8,28 & 8,00 & 8,41 & 8,31 & 8,54 & 8,48 & 8,66 & 8,70 & 8,92 & 8,79 & 9,08 & 8,84 & 9,45 & 8,94 & 9,64 & 9,10 & 10,35 & 9,86 \\
\hline LI & 5,44 & 5,47 & 6,14 & 6,04 & 6,29 & 6,23 & 6,54 & 6,28 & 6,71 & 6,45 & 6,84 & 6,55 & 6,92 & 6,72 & 7,23 & 6,96 & 7,45 & 7,19 & 7,68 & 7,33 & 8,00 & 7,76 \\
\hline C &, 92 & 6,94 & 7,34 & 7,07 & 7,63 & 7,31 & 7,76 & 7,43 & 7,91 & 7,53 & 7,99 & 7,64 & 8,04 & 7,81 & 8,11 & 7,87 & 8,44 & 7,99 & 8,72 & 8,14 & 9,16 & 8,49 \\
\hline 1PM & 6,36 & 6 , & 6,67 & 6,65 & 6,88 & 6,89 & 7,07 & 6,96 & 7,23 & 7 & 7,34 & 10 & 7,43 & 16 & 7,55 & 7,30 & 7,65 & 7,39 & 7,92 & 7,52 & 8,50 & 8,22 \\
\hline 2PM & 6,08 & & 6,42 & & 6,56 & & 6,69 & 6,67 & 6,79 & & & & 7,11 & & 7,20 & 7 & 7,40 & 7,16 & 7,54 & 7,25 & 7,84 & 7,60 \\
\hline $1 \mathrm{M}$ & 9,31 & 9,03 & 9,76 & 9,48 & 10,10 & 9,64 & 10,27 & 9,76 & 10,38 & 10,03 & 10,49 & 10,25 & 10,62 & 10,37 & 10,82 & 10,48 & 10,92 & 10,66 & 11,08 & 10,84 & 12,10 & 12,42 \\
\hline \multicolumn{23}{|c|}{ MB Arch } \\
\hline $\mathrm{Cl}$ & 4,81 & 4,72 & 5,08 & 5,05 & 5,25 & 5,16 & 5,38 & 5,29 & 5,45 & 5,48 & 5,53 & 5,53 & 5,61 & 5,60 & 5,74 & 5,72 & 5,80 & 5,90 & 5,97 & 6,06 & 6,18 & 6,20 \\
\hline LI & 5,25 & 4,83 & 5,44 & 5,47 & 5,56 & 5,65 & 5,68 & 5,77 & 5,90 & 5,93 & 6,10 & 6,02 & 6,18 & 6,07 & 6,28 & 6,14 & 6,47 & 6,27 & 6,63 & 6,48 & 7,02 & 6,77 \\
\hline C & 6,28 & 5,66 & 6,42 & 6,17 & 6,69 & 6,33 & 6,84 & 6,53 & 6,96 & 6,64 & 7,01 & 6,66 & 7,10 & 6,84 & 7,24 & 6,94 & 7,47 & 7,03 & 7,67 & 7,20 & 8,05 & 7,39 \\
\hline 1PM & 6,26 & 6,27 & 6,67 & 6,48 & 6,85 & 6,70 & 7,12 & 6,82 & 7,24 & 6,90 & 7,34 & 6,99 & 7,43 & 7,14 & 7,47 & 7,25 & 7,58 & 7,44 & 7,72 & 7,55 & 8,08 & 7,81 \\
\hline 2PM & 6,56 & 6,44 & 6,89 & 6,69 & 7,05 & 6,86 & 7,27 & 7,06 & 7,41 & 7,18 & 7,51 & 7,30 & 7,60 & 7,41 & 7,78 & 7,52 & 7,88 & 7,59 & 8,06 & 7,80 & 8,29 & 8,03 \\
\hline $1 \mathrm{M}$ & 10,03 & 10,15 & 10,53 & 10,38 & 10,71 & 10,68 & 10,83 & 10,87 & 11,09 & 10,96 & 11,42 & 11,15 & 11,55 & 11,35 & 11,75 & 11,58 & 11,88 & 11,83 & 12,09 & 11,99 & 13,03 & 12,26 \\
\hline
\end{tabular}

Table 3. Mesiodistal tooth-size table (percentiles) for adults (male-M and female- $\neg$ F) for both maxillary (MX) and mandibular (MB) arches. CI-central incisor; LI-lateral incisor; C-canine; 1PM-1rst premolar; 2PM-2nd premolar; 1M-1rst molar.

\begin{tabular}{|c|c|c|c|c|c|c|c|c|c|c|c|c|c|c|c|c|c|c|c|c|c|c|}
\hline \multirow{3}{*}{ TEETH } & \multicolumn{6}{|c|}{ Small } & \multicolumn{10}{|c|}{ Average } & \multicolumn{6}{|c|}{ Large } \\
\hline & \multicolumn{2}{|c|}{ Min. } & \multicolumn{2}{|c|}{10} & \multicolumn{2}{|c|}{20} & \multicolumn{2}{|c|}{30} & \multicolumn{2}{|c|}{40} & \multicolumn{2}{|c|}{50} & \multicolumn{2}{|c|}{60} & \multicolumn{2}{|c|}{70} & \multicolumn{2}{|c|}{80} & \multicolumn{2}{|c|}{90} & \multicolumn{2}{|c|}{ Máx. } \\
\hline & $\mathrm{M}$ & $\mathrm{F}$ & $\mathrm{M}$ & & $\begin{array}{ll}\mathrm{M} \\
\end{array}$ & $\mathrm{F}$ & M & & $\mathrm{M}$ & $F$ & \begin{tabular}{l|l}
$\mathrm{M}$ \\
\end{tabular} & & $\mathrm{M}$ & $\mathrm{F}$ & $\mathrm{M}$ & & $\mathrm{M}$ & $F$ & $\mathrm{M}$ & & $\mathrm{M}$ & $\mathrm{F}$ \\
\hline \multicolumn{23}{|c|}{ MX Arch } \\
\hline Cl & 7,31 & 7,66 & 7,93 & 7,91 & 8,05 & 8,09 & 8,31 & 8,26 & 8,47 & 8,40 & 8,69 & 8,54 & 8,82 & 8,66 & 8,97 & 8,78 & 9,13 & 8,97 & 9,28 & 9,25 & 9,76 & 9,78 \\
\hline LI & 5,36 & 5,46 & 6,01 & 5,72 & 6,34 & 6,25 & 6,47 & 6,42 & 6,59 & 6,52 & 6,76 & 6,65 & 6,81 & 6,76 & 6,94 & 6,89 & 7,18 & 6,97 & 7,34 & 7,12 & 7,82 & 8,38 \\
\hline C & 6,94 & 6,81 & 7,40 & 7,09 & 7,67 & 7,38 & 7,86 & 7,51 & 7,92 & 7,63 & 7,99 & 7,78 & 8,03 & 7,82 & 8,11 & 7,94 & 8,31 & 8,05 & 8,49 & 8,34 & 8,85 & 8,86 \\
\hline 1PM & 6,49 & 6,26 & 6,78 & 6,55 & 6,94 & 6,72 & 7,11 & 6,91 & 7,18 & 7,05 & 7,26 & 7,13 & 7,33 & 7,18 & 7,44 & 7,30 & 7,56 & 7,37 & 7,64 & 7,58 & 8,12 & 7,89 \\
\hline 2PM & 6,04 & 5,98 & 6,57 & 6,29 & 6,65 & 6,44 & 6,82 & 6,64 & 6,95 & 6,82 & 7,01 & 6,91 & 7,18 & 7,05 & 7,24 & 7,19 & 7,36 & 7,27 & 7,55 & 7,38 & 7,93 & 7,77 \\
\hline $1 \mathrm{M}$ & 9,55 & 9,31 & 9,88 & 9,64 & 10,14 & 9,94 & 10,27 & 10,08 & 10,41 & 10,20 & 10,55 & 10,24 & 10,60 & 10,37 & 10,74 & 10,51 & 10,92 & 10,69 & 11,21 & 10,95 & 12,05 & 11,58 \\
\hline \multicolumn{23}{|c|}{ MB Arch } \\
\hline $\mathrm{Cl}$ & 4,21 & 4,60 & 5,08 & 4,97 & 5,22 & 5,08 & 5,27 & 5,19 & 5,38 & 5,30 & 5,45 & 5,41 & 5,54 & 5,49 & 5,67 & 5,57 & 5,74 & 5,69 & 5,94 & 5,92 & 6,27 & 6,24 \\
\hline LI & 4,68 & 5,03 & 5,48 & 5,41 & 5,66 & 5,58 & 5,76 & 5,69 & 5,80 & 5,76 & 5,92 & 5,81 & 6,01 & 5,90 & 6,09 & 5,96 & 6,27 & 6,13 & 6,50 & 6,36 & 6,81 & 7,14 \\
\hline C & 6,24 & 5,72 & 6,49 & 6,27 & 6,66 & 6,37 & 6,84 & 6,47 & 6,99 & 6,58 & 7,06 & 6,74 & 7,16 & 6,81 & 7,27 & 6,96 & 7,35 & 7,06 & 7,45 & 7,25 & 7,73 & 7,60 \\
\hline 1PM & 6,20 & 6,31 & 6,74 & 6,63 & 6,93 & 6,81 & 7,09 & 6,97 & 7,20 & 7,02 & 7,29 & 7,15 & 7,39 & 7,27 & 7,49 & 7,37 & 7,59 & 7,55 & 7,76 & 7,69 & 8,54 & 8,06 \\
\hline 2PM & 6,42 & 6,33 & 6,93 & 6,71 & 7,08 & 6,99 & 7,21 & 7,08 & 7,32 & 7,18 & 7,47 & 7,27 & 7,58 & 7,44 & 7,62 & 7,52 & 7,76 & 7,71 & 7,92 & 7,82 & 8,40 & 8,15 \\
\hline $1 \mathrm{M}$ & 9,82 & 9,65 & 10,53 & 10,43 & 10,84 & 10,59 & 11,01 & 10,75 & 11,18 & 10,90 & 11,38 & 11,11 & 11,48 & 11,28 & 11,68 & 11,39 & 11,83 & 11,51 & 12,09 & 11,81 & 12,76 & 12,44 \\
\hline
\end{tabular}

\section{Discussion}

Although there are many tooth-size tables existing in the literature, we have not found any ordered by percentiles for a Spanish population. The only tables ordered by percentiles are those of Sanin and Savara (7) for an American population. These are not divided by age groups as ours are, but presented as a whole, without specifying the age of the sample, although by the clinical cases presented and the sample characteristics described in Sanin and Savara's manuscript (7), we can assume that it is made up of adolescents. Just as with those authors, our study considered the mean distance between both semi-arches for each tooth, once symmetry had been observed between contralateral teeth.

First of all, we compared the dental-size tables of our three age groups.

In our study, we found that both gender and age influence mesiodistal tooth size. However, whereas in young adults and adults sexual dimorphism was statistically significant $(p<0.01$ and $p<0.02$ respectively), males presenting greater diameters than females, in the adoles- cent group mesiodistal tooth size diameters presented no statistical differences between genders. Such sexual dimorphism of mesiodistal tooth size is well documented in the literature where, in general, adolescents also present significant differences per gender, contrary to our results $(2,3)$.

We observed that adolescent mesiodistal tooth sizes were greater than those of young adults and adults, although this difference was very slight in males, with a mean value of $0,15 \mathrm{~mm}$ between adolescents and adults with a statistically significant difference. This implies a progressive decrease $(0.11 \mathrm{~mm})$ that is significant between adolescents and young adults. Despite that, studying each tooth individually, we only found statistically significant differences for the upper lateral incisor $(0.33 \mathrm{~mm})$ between adolescents and adults.

For females, the mean decrease of mesiodistal tooth size with age is greater than for males, $0.32 \mathrm{~mm}$ between adolescents and adults, and between adolescents and young adults. On observing the decrease for each tooth between adolescents and adults, we found significant 
decreases $(p<0.05)$ for all teeth except for the upper second premolar. These significant differences varied from $0,47 \mathrm{~mm}$ for the upper lateral incisor to $0,21 \mathrm{~mm}$ for the lower second premolar. The comparison between adolescents and young adults showed that, in this case, the reduction was not significant for either the upper second premolar, the lower first molar or the lower central incisor. The differences varied from $0,55 \mathrm{~mm}$ for the upper first molar to $0,24 \mathrm{~mm}$ for the lower second premolar.

This would seem to indicate that, in both genders, there is a decrease in the mesiodistal tooth size with age that seems to be more pronounced between the adolescent and young adult groups, which continues to decrease but at a slower rate between the young adult and adult groups. Furthermore, whereas the decrease is small in males, there is a greater and statistically more significant decrease in females.

This decrease in mesiodistal tooth size with age in contemporary western individuals is insufficiently documented in the literature, being found mainly in studies of previous civilizations or in non-western populations with different kinds of food that require more vigorous chewing, which in turn causes much greater tooth wear. As an example of this, Begg's (9) studies on Australian Aborigines from the Stone Age show 10,54mm of mesiodistal tooth size reduction for the lower arch and Kieser et al. (10) observed an increase of the interproximal posterior tooth contact surface with age as a consequence of attrition in Paraguayan Indians. These mean values of interproximal tooth reduction in the different age groups were much greater than those observed in our study.

It would appear logical to affirm that teeth most probably tend to decrease with age in mesiodistal size due to interproximal wear. With regard to the difference between the genders of our results, it should be borne in mind that our study is not a longitudinal study on the same sample, but is a sample consisting of three different age groups of patients, and that, therefore, the explanation for this phenomenon did not form part of this investigation.

Finally, comparing the results of our adolescent sample with those of Sanin and Savara (7), our tables on a Spanish population, generally speaking, present values slightly higher than those of those authors, especially for the women, but once again it should be borne in mind that we do not know the age of their study sample and, therefore, we cannot accurately compare their results with ours.

Although mesiodistal tooth size tables ordered by percentiles are presented separately by gender for our sample of adolescents, no statistically significant differences were found between them, unlike the Sanin and Savara tables (7) where such differences exist. Graphics have been made for all percentiles between the two groups but Figure 1 is presented as an example for compa- ring the tooth sizes of our sample with those of Sanin and Sanin and Savara's for the minimum (0), medium $(50,60)$ and maximum $(100)$ percentiles. We have chosen these middle percentiles because they are the most common teeth sizes. In these graphs, we can see how the lines corresponding to the mesiodistal sizes of men and women in our sample almost overlap, while Sanin and Savara sizes are smaller than ours with the lines of men and women quite separate.

We believe that it is of great interest to have this type of dental table, organised by percentiles, in order to evaluate the tooth size of an individual that belongs to a certain population group. However, we also believe that it is important for future studies to take a longitudinal approach and to include a larger sample so as to better appreciate the results.

\section{Conclusions}

- Three mesiodistal tooth-size tables, organized by percentiles, in a Spanish population sample were drawn up distinguishing between ages (adolescents, young adults and adults) and gender.

- The mesiodistal tooth size of adolescents did not present statistical differences between genders in the lists of percentiles, contrary to the other age groups.

- Mesiodistal tooth size diminishes with age, more markedly in women.

- The values obtained were slightly higher to those found by Sanin and Savara in an American population, especially for women.

\section{References}

References with links to Crossref - DOI

1. Puri N, Pradhan KL, Chandna A, Sehgal V, Gupta R. Biometric study of tooth size in normal, crowded, and spaced permanent dentitions. Am J Orthod Dentofacial Orthop. 2007;132:279.e7-14.

2. Bishara SE, Jakobsen JR, Abdallah EM, Fernandez Garcia A. Comparisons of mesiodistal and buccolingual crown dimensions of the permanent teeth in three populations from Egypt, Mexico, and the United States. Am J Orthod Dentofacial Orthop. 1989;96:416-22.

3. Haralabakis NB, Sifakakis I, Papagrigorakis M, Papadakis G. The correlation of sexual dimorphism in tooth size and arch form. World J Orthod. 2006;7:254-60.

4. Keene HJ. Mesiodistal crown diameters of permanent teeth in male American Negroes. Am J Orthod. 1979;76:95-9.

5. Lavelle CL. Maxillary and mandibular tooth size in different racial groups and in different occlusal categories. Am J Orthod. 1972;61:29-37.

6. Townsend GC. Tooth size in children and young adults with trisomy 21 (Down) syndrome. Arch Oral Biol. 1983;28:159-66.

7. Sanin C, Savara BS. An analysis of permanent mesiodistal crown size. Am J Orthod. 1971;59:488-500.

8. Paredes V, Gandia JL, Cibrian R. New, fast, and accurate procedure to calibrate a 2-dimensional digital measurement method. Am J Orthod Dentofacial Orthop. 2005;127:518-9.

9. Corruccini RS. Australian aboriginal tooth succession, interproximal attrition, and Begg's theory. Am J Orthod Dentofacial Orthop. 1990;97:349-57.

10. Kieser JA, Groeneveld HT, Preston CB. Patterns of dental wear in the Lengua Indians of Paraguay. Am J Phys Anthropol. 1985;66:21-9. 